\title{
A biomechanical analysis of the stand-up paddle board stroke: A comparative study
}

\author{
Ben Schram $^{1,2}$, James Furness ${ }^{1,2}$, Kevin Kemp-Smith ${ }^{\text {Corresp., 1, } 2 \text {, Jason Sharp }}{ }^{2}$, Matt Christini ${ }^{2}$, Daniel Harvie ${ }^{2}$, \\ Emma Keady $^{2}$, Maichel Ghobrial ${ }^{2}$, Josh Tussler ${ }^{2}$, Wayne Hing ${ }^{1,2}$, Jeff Nessler ${ }^{3}$, Matt Becker $^{3}$ \\ ${ }^{1}$ Water Based Research Unit, Bond Institute of Health and Sport, Bond University, Robina, Queensland, Australia \\ 2 Faculty of Health Science and Medicine, Bond Institute of Health and Sport, Bond University, Robina, Queensland, Australia \\ 3 Department of Kinesiology, California State University, San Marcos, San Marcos, California, United States \\ Corresponding Author: Kevin Kemp-Smith \\ Email address: kkempsmi@bond.edu.au
}

Background: Stand-up paddle boarding (SUP) is a rapidly growing global aquatic sport, with increasing popularity among participants within recreation, competition and rehabilitation. To date, few scientific studies have focused on SUP. Further, there is no research examining the biomechanics of the SUP paddle stroke. The purpose of this study was to investigate whether variations in kinematics existed among experienced and inexperienced SUP participants using three-dimensional motion analysis. This data could be of significance to participants, researchers, coaches and health practitioners to improve performance and inform injury minimization strategies.

Methods: A cross sectional observational design study was performed with 7 experienced and 19 inexperienced paddlers whereby whole-body kinematic data were acquired using a 6-camera Vicon motion capture system. Participants paddled a on SUP ergometer while three-dimensional range of motion (ROM) and peak joint angles were calculated for the shoulders, elbows, hips and trunk. Mann Whitney $U$ tests were conducted on the non-normally distributed data to evaluate differences between level of expertise.

Results: Significant differences in joint kinematics were found between experienced and inexperienced participants, with inexperienced participants using greater overall shoulder range of motion (ROM) (78.9 \pm 24.9 o vs $\left.56.6 \pm 17.3^{\circ}, p=0.010\right)$ and less hip ROM than the experienced participants $\left(50.0 \pm 18.5^{\circ}\right.$ vs $\left.66.4 \pm 11.8^{\circ} p=0.035\right)$. Experienced participants demonstrated increased shoulder motion at the end of the paddle stoke compared to the inexperienced participants $(74.9 \pm 16.30$ vs $35.2 \pm 28.50 p=0.001$ minimum shoulder flexion) and more extension at the elbow (6.0 $9.2^{\circ}$ minimum elbow flexion vs $24.8 \pm$ $13.5^{\circ} p=0.000$ ) than the inexperienced participants.

Discussion: The results of this study indicate several significant kinematic differences between the experienced and inexperienced SUP participants. These variations in technique were noted in the shoulder, elbow and hip and are evident in other aquatic paddling sports where injury rates are higher in these joints. These finding may be valuable for coaches, therapists and participants needing to maximise performance and minimize injury risk during participation in SUP. 


\section{A Biomechanical Analysis of the Stand-Up Paddle Board Stroke: A Comparative Study}

2 Ben Schram $(\mathrm{PhD})^{1,2}$, James Furness $(\mathrm{PhD})^{1,2}$, Kevin Kemp-Smith (ScD) 1,2, Jason Sharp

3 (BExSci $^{2}{ }^{2}$, Matt Christini (BExSci) ${ }^{2}$, Daniel Harvey $(\mathrm{PhD})^{2}$, Emma Keady (BExSci) ${ }^{2}$, Maichel

4 Ghobrial (BExSci) ${ }^{2}$, Josh Tussler $(\mathrm{BSci})^{2}$, Wayne Hing (PhD) ${ }^{1,2}$, Jeff Nessler (PhD) ${ }^{3}$, Matt

5 Becker $(\mathrm{MSc})^{3}$

$6{ }^{1}$ Water Based Research Unit, Bond Institute of Health and Sport, Bond University QLD,

$7 \quad$ Australia

$8 \quad{ }^{2}$ Faculty of Health Science and Medicine, Bond Institute of Health and Sport, Bond

9 University, QLD, Australia.

$10{ }^{3}$ California State University, San Marcos, CA, United States of America

\section{Corresponding Author:}

13 Kevin Kemp-Smith ${ }^{1,2}$

14 Bond Institute of Health and Sport, 2 Promethean Way, Robina, Queensland, 4226, Australia. 


\section{Abstract}

Background: Stand-up paddle boarding (SUP) is a rapidly growing global aquatic sport, with increasing popularity among participants within recreation, competition and rehabilitation. To date, few scientific studies have focused on SUP. Further, there is no research examining the biomechanics of the SUP paddle stroke. The purpose of this study was to investigate whether variations in kinematics existed among experienced and inexperienced SUP participants using three-dimensional motion analysis. This data could be of significance to participants, researchers, coaches and health practitioners to improve performance and inform injury minimization strategies.

Methods: A cross sectional observational design study was performed with 7 experienced and 19 inexperienced paddlers whereby whole-body kinematic data were acquired using a 6-camera Vicon motion capture system. Participants paddled a on SUP ergometer while three-dimensional range of motion (ROM) and peak joint angles were calculated for the shoulders, elbows, hips and trunk. Mann Whitney U tests were conducted on the non-normally distributed data to evaluate differences between level of expertise.

34 Results: Significant differences in joint kinematics were found between experienced and inexperienced participants, with inexperienced participants using greater overall shoulder range of motion $(\mathrm{ROM})\left(78.9 \pm 24.9^{\circ}\right.$ vs $\left.56.6 \pm 17.3^{\circ}, p=0.010\right)$ and less hip ROM than the experienced participants $\left(50.0 \pm 18.5^{\circ}\right.$ vs $\left.66.4 \pm 11.8^{\circ} p=0.035\right)$. Experienced participants demonstrated increased shoulder motion at the end of the paddle stoke compared to the inexperienced participants 
$39\left(74.9 \pm 16.3^{\circ}\right.$ vs $35.2 \pm 28.5^{\circ} p=0.001$ minimum shoulder flexion $)$ and more extension at the elbow

$40\left(6.0 \pm 9.2^{\circ}\right.$ minimum elbow flexion vs $\left.24.8 \pm 13.5^{\circ} p=0.000\right)$ than the inexperienced participants.

41 Discussion: The results of this study indicate several significant kinematic differences between

42 the experienced and inexperienced SUP participants. These variations in technique were noted in

43 the shoulder, elbow and hip and are evident in other aquatic paddling sports where injury rates are

44 higher in these joints. These finding may be valuable for coaches, therapists and participants

45 needing to maximise performance and minimize injury risk during participation in SUP.

\section{Introduction:}

48

Stand-up paddle boarding (SUP) is a mixture of surfing and paddle-based sports where the rider balances on a board ( $\sim 3-5$ meters long, $\sim 1$ meter wide) and grips a single-bladed paddle ( $\sim 2$ meters long) to propel themselves through the water (Schram 2015). Previous research has defined the main components of the SUP stroke as; entry, drive and exit of the paddle from the water. The entry phase denotes entry of the paddle into the water, the drive phase is the forceful pulling stroke through the water and exit phase describes the paddle release and withdrawal from the water (Schram et al. 2015). To date there has been no scientific research analysing the biomechanics of the paddle stroke in SUP.

SUP is an aquatic recreational and sporting activity that is readily accessible to most people, requires minimal equipment, is easy to learn and provides a low impact physical challenge (Schram 2015). Despite being a relatively new water-based sport, SUP's popularity has increased globally due to its purported health and fitness benefits such as improvements in fitness, endurance and 
61 self-rated quality of life (Schram et al. 2016b). According to the '2015 Paddlesports Report', SUP

62 participation has steadily increased in the United States from 1.1 million in 2010 to 2.8 million in

632014 (Outdoor Foundation and The Coleman Company, 2015). SUP is an activity that is suitable

64 for all ages and skill levels, can be practiced on any body of water and is reported to be an ideal

65 activity for a full-body workout (Mei-Dan \& Carmont 2013; Schram et al. 2017).

66

67 Biomechanical analysis in sport allows for modifications to technique in order to maximise power

68 output and minimise injury (Bini \& Carpes 2014; Ho et al. 2009). Epidemiological studies of

69 injuries in SUP have revealed that the shoulder/upper arm (32.9\%) lower back (14.3\%) and

70 elbow/forearm (11.8\%) were the most common locations of injuries reported in a study of both

71 competitive and recreational SUP riders (Furness et al. 2017). The importance of technique is

72 highlighted by the fact that less than optimal stroke biomechanics has been associated with both

73 shoulder, elbow and back injuries in the similar sports of kayaking, and outrigger paddling

74 (Hagemann et al. 2004). In line with epidemiological studies in SUP, the shoulder is also the most

75 commonly injured site in kayaking accounting for in excess of $30 \%$ of all paddling injuries

76 (Abraham \& Stepkovitch 2012; Bell et al. 2013; Fiore \& Houston 2001). A biomechanical

77 understanding of the SUP stroke may provide direction towards injury minimisation within this

78 sport.

Currently, only non-scientific information exists regarding optimal paddling technique for SUP in

81 the form of online media and instructional videos (Cain 2015a; Dionne 2014; Stehlik 2011), and written guides (Cain 2015b; Wordpress. 2015). Research into stroke biomechanics has been performed in similar aquatic sports including kayaking, canoeing, and dragon boat racing. 
84 However, these studies focused on comparisons between skill levels (Ho et al. 2009; Kendal \&

85 Sanders 1992; Limonta et al. 2010a), genders (Gomes et al. 2015), equipment (Fleming et al.

86 2012), training paces (Gomes et al. 2015; Zahalka et al. 2011) and dominant vs non-dominant sides

87 (Limonta et al. 2010b; Wassinger et al. 2011). The purpose of these previous investigations was

88 to determine mechanisms in which to maximise performance and minimize injury risk. Despite

89 sharing similarities to other aquatic paddling sports, the SUP stroke does have considerable

90 biomechanical differences. Primarily, the participant is standing up and balancing on a board

91 compared to all other paddle sports where the participant is sitting. Therefore, the purpose of this

92 study was to compare the differences in SUP paddle stroke kinematics between experienced and

93 inexperienced participants. The hypothesis is that there will be significant differences between

94 experience levels of paddlers in regard to paddling technique. Findings may assist in identifying

95 optimal stroke mechanics in order to minimize injury occurrence and improve overall

96 performance. 
98

99

100

101

102

103

104

105

106

107

108

109

110

111

112

113

114

115

116

117

118

119

\section{Materials \& Methods}

\section{Participants}

Experienced and inexperienced SUP participants were recruited for the study. Exclusion criteria included a history of current musculoskeletal injuries or cardiovascular disorders that impacted their ability to undertake the trials. Additionally, any participant that had an allergy to adhesive tape was also excluded. Recruitment was conducted through flyers, emails, and face-to-face requests with information to participate in a within-participant laboratory biomechanical analysis of the SUP paddle stroke. An explanatory statement was provided to potential participants and a consent form was provided to those interested in being involved in the study. To be classified as experienced, participants were to have had a history of competition at an international, national or state level within the previous two years. Participants who engaged in SUP recreationally and had no history of competition of formal training were classified as inexperienced.

In total, twenty-six SUP participants were recruited (experienced $n=7,4$ male, 3 female, $33 \pm 7.8 \mathrm{yrs}$, $173.9 \pm 50.5 \mathrm{~cm}, \quad 76.5 \pm 12.2 \mathrm{~kg}$; inexperienced $\mathrm{n}=19,11$ male, eight female, $24.5 \pm 2.4 \mathrm{yrs}$, $174.1 \pm 63.3 \mathrm{~cm}, 72.9 \pm 11.3 \mathrm{~kg}$ ) for this study. The experienced paddlers, on average trained 5 times per week on water and twice on land. Participants were invited to attend a single data collection session at the Bond Institute of Health Motion Analysis Laboratory. Permission to conduct the study was granted by the University Human Research Ethics Committee (0000015422) and all participants provided written informed consent prior to participation.

\section{General Protocol}


120 A 6-camera, passive, three-dimensional motion analysis system at $100 \mathrm{~Hz}$ (Vicon; Oxford

121 Metrics, Inc.) was utilized to track $1 \mathrm{~cm}$ spherical retroflective markers placed over key bony

122 landmarks according to Vicon's full body Plug-In Gait model. This model uses 39 reflective

123 markers $(14 \mathrm{~mm})$ diameter to create nine segments. Cameras were strategically placed around the

124 test area to maximize data capture. Prior to data acquisition, the motion capture system was

125 calibrated in accordance with manufacturer recommendations (Vicon; Oxford Metrics, Inc.)

126 whereby an L-frame calibration wand was used to align the origin of the capture volume with a

127 point on the surface of a specialised SUP ergometer (KayakPro SUPErgo, Miami, FL, USA).

128 The KayakPro SUP ergometer has previously been validated for clinical testing (Schram et al.,

129 2015). A static trial was undertaken for anatomical landmark calibration for each of the

130 participants (Besier 2003).

131

132 Participants undertook a familiarization period prior to testing which involved a 2-minute warm

133 up where they self-selected stroke frequency, stance, rate and paddle change over to the opposite

134 side. At completion of the familization period, the participant performed two consecutive

135 paddling trials (left and right side), in a randomised fashion predetermined by a spreadsheet

136 formula (Microsoft Excel v16.0, Washington, USA). During each trial, participants were

137 instructed to maintain a power output of $20 \mathrm{~W}$ for a total of 40 seconds. This was considered to

138 be a moderate paddle intensity based on previous studies (Schram et al. 2016c).

140 Vicon data were visually inspected and labelled using Vicon Nexus 2.5 (Nexus; Oxford Metrics,

141 Inc.). Small gaps were filled using a built-in spline interpolation function with larger gaps filled 142 using the pattern fill function (based upon the closest available anatomical landmark). Raw data 
143 files were exported from Nexus and analyzed in Visual3D (C-Motion, Germantown, MD, USA).

144 Kinematic variables of interest included peak and minimum joint angles of the shoulder, elbow,

145 lumbar spine and hip. Joint angle time series data were then analyzed using custom routines

146 written in MATLAB (R2015b, Natick, MA). The beginning and end of each stroke was defined

147 as the maximum anterior position of the right hand on the right side and the left hand while

148 paddling on the left side. These positions were taken from the raw marker data, and their respective

149 times were then used to analyse joint angles. Range of motion, peak and minimum joint angles

150 were then calculated from the mean stroke profile for each joint of interest. The shoulder, elbow,

151 lumbar and hip mean joint angles were obtained by paddling on the left and right side and were

152 then averaged together to generate a single profile of motion at the joint. This was achieved by

153 combining the respective ipsilateral and contralateral angles (e.g. averaging the right shoulder

154 during right side paddling with the left shoulder during left side paddling). The time series data

155 were filtered ( $4^{\text {th }}$ order Butterworth low pass, $20 \mathrm{~Hz}$ cut off) and averaged across each participants'

156 strokes. These average joint angle trajectories were plotted for comparison between levels of

157 experience.

158

159

Data Analysis

160 Descriptive statistics were calculated including means, standard deviation and coefficient of 161 variance for each joint. Data were found to be not equally distributed in a Shapiro-Wilks test and 162 therefore, Mann Whitney $U$ tests were conducted to determine differences between groups. Effect 163 sizes were calculated for all comparisons to reflect the magnitude of change. The effect size was 164 calculated using the method developed by Clark-Carter where the $\mathrm{z}$ value derived from the Mann165 Whitney $U$ Test is converted to into an $\mathrm{r}$ value (Clark-Carter 2018). Consequently, the $\mathrm{r}$ value can 
166 be interpreted using the classification developed by Cohen, where an effect size of 0.1 could be

167 considered small, 0.3 could be considered medium and 0.5 could be considered large (Cohen

168 2013). Statistical significance was set at $p=0.05$ and all statistical analyses were completed using

169 the IBM Statistical Package for the Social Sciences (SPSS) v24.0 (SPSS, Inc., Chicago, Illinois, 170 USA).

171

\section{Results}

173 Experienced participants were found to be on average 9 years older $(\mathrm{p}<0.001)$ than inexperienced

174 participants. There were no other significant differences in height or weight between the groups.

175 Table 1 shows the overall range of motion (ROM), maximum and minimum joint angles for each

176 for the assessed joints.

177

178 Table 1: Here

179

Inexperienced participants demonstrated a significantly $(p=0.010)$ greater overall range of

181 motion in the shoulder compared with the experienced participants $\left(78.9^{\circ} \pm 24.9^{\circ}\right.$ vs $56.6^{\circ} \pm$ $17.3^{\circ}$ respectively); resulting in a $39.4 \%$ difference. Consequently, the minimum shoulder angle was significantly ( $p=0.001)$ lower within the inexperienced participants compared with the experienced participants $\left(35.2^{\circ} \pm 28.5^{\circ}\right.$ vs. $74.9^{\circ} \pm 16.3^{\circ}$ respectively); resulting in a $53 \%$ difference. 
186 During hip flexion, the experienced participants demonstrated a significantly $(p=0.035)$ greater

187 total range of motion compared with the inexperienced participants $\left(66.4^{\circ} \pm 11.8^{\circ}\right.$ vs $50.0^{\circ} \pm$

$18818.5^{\circ}$ respectively); resulting in $24.7 \%$ difference.

189 During elbow flexion the minimum angle was significantly $(p=0.000)$ less within the

190 experienced participants $\left(6.0^{\circ} \pm 9.2^{\circ}\right.$ vs $24.8^{\circ} \pm 13.5$ respectively); resulting in a $75.8 \%$

191 difference.

192

193 Figure 1 displays these differences graphically. The experienced paddler is seen to display more

194 hip flexion during the three stroke phases and less elbow flexion.

195

196

197

198

199

200

201

202

203

204

205

206

207

\section{Figure 1: Here}

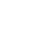

\section{Discussion}

To our knowledge, this is the first known study to examine the stroke kinematics of SUP. The purpose of this research was to compare the differences in stroke kinematics between experienced and inexperienced participants. The results conclude that important differences exist in the paddling technique of both experienced and inexperienced participants, specifically at the shoulder, elbow and hip.

Previous research examining different skill levels in dragon boat racing found no differences in stroke kinematics between elite and sub-elite participants (Ho et al. 2009). In that study both the elbow and shoulder were examined during the entry, drive and exit phases of the stroke. It should be noted however, the reference group in the study were sub-elite experienced participants and not 
208 the inexperienced participants utilised in the current study. Paddling kinematics in the study

209 highlighted within the reference group, $103^{\circ}$ of elbow ROM and approximately $140^{\circ}$ of shoulder

210 ROM throughout the stroke cycle (Ho et al. 2009). Kinematic investigations of the kayak stroke

211 have also reported elbow ROM in the order of $100^{\circ}$ during the paddle stroke cycle amongst a

212 variety of skill levels (Limonta et al. 2010b). The fact that SUP is performed in a standing position

213 would negate the need for larger shoulder and elbow ROM, highlighted by increased trunk flexion

214 in the experienced group. This is thought to be a strategy to increase stroke length among

215 experienced participants, who have previously been reported to have a longer, more powerful 216 stroke than their more novice counterparts (Schram et al. 2016c).

217

218 Overall, inexperienced participants displayed greater overall total shoulder ROM and less total

219 hip flexion ROM while paddling. The reduced hip motion, combined with greater shoulder

220 movement, illustrates a tendency for the inexperienced group to rely heavily on the shoulder and

221 possibly the biceps and forearm musculature to generate force during the entry and drive phases

222 of the stroke. It is unclear at this stage whether this may predispose the shoulder and elbow to

223 injury, both of which were shown to be a common site of injury in SUP paddlers (Furness et al.

224 2017). In contrast, the experienced participants had less overall shoulder ROM and greater hip

225 ROM. Interestingly, experienced participants initiated and ended the entry phase at a greater

226 shoulder flexion angle, likely reflective of the greater hip flexion and a more horizontal trunk at

227 the point of entry. Further, data indicated significantly less minimum elbow flexion in the

228 experienced group, indicating the experienced participants were more likely to enter and drive

229 through the stroke with an extended arm. 
231 In summary, these data suggest that experienced participants rely less on shoulder and minimum

232 elbow ROM but employ more hip flexion ROM. This would suggest a strategy facilitating an

233 increased reaching motion before the initial paddle entry - a finding which may be of

234 significance when considering the shoulder and elbow joints as injury prone regions in SUP

235 participants (Furness et al. 2017).

\section{Study limitations}

238

239

240

241

242

243

244

245

246

247

248 
253 body (Kendal \& Sanders 1992) and future studies should also consider this variable. Finally, the

254 inexperienced participants analysed represented a wide range of experience levels, ranging from

255 minimal exposure to SUP to six months experience at a recreational level. This led to a largely

256 heterogenous group for the inexperienced participants. Some of the inexperienced participants

257 also had difficulty maintaining the required power output for the duration of the assessments,

258 consequently, differences in workload among the participants may have also affected the

259 analysed kinematics.

260

261

262

263

Conclusions

264 The results of this study suggest there are significant differences in paddle stroke kinematics

265 between experienced and inexperienced SUP participants. Inexperienced participants appear to

266 be more reliant on larger ranges of motion at the shoulder joint and less hip motion. Experienced

267 participants appear to utilise less total shoulder range of motion and more overall hip range of

268 motion. Identifying these different kinematic strategies may be of benefit for coaches,

269 rehabilitation professionals and participants interested in improving technique and minimizing

270 injury risk.

271

272 References 
273 Abraham D, and Stepkovitch N. 2012. The Hawkesbury Canoe Classic: Musculoskeletal Injury

274

275

276

277

278

279

280

281

282

283

284

285

286

287

288

289

290

291

292

293

294

295

296

297

Surveillance and Risk Factors Associated With Marathon Paddling. Wilderness \& Environmental Medicine 23:133-139. 10.1016/j.wem.2012.02.014

Bell R, Carman A, and Tumilty S. 2013. Sports injury profile of competitive Waka Ama (outrigger canoe) paddlers in New Zealand. New Zealand Journal of Physiotherapy 41:30-35.

Besier TF, Sturnieks, D. L., Alderson, J. A., \& Lloyd, D. G. 2003. Repeatability of gait data using a functional hip joint centre and a mean helical knee axis. Journal of biomechanics 36:1159-1168.

Bini RR, and Carpes FP. 2014. Introduction to biomechanical analysis for performance enhancement and injury prevention. In Biomechanics of Cycling.: Springer.

Cain L. 2015a. "Big Picture" Approach to Technique Part 3 - Using Big Muscles and Body Weight.

Cain L. 2015b. How to Improve Your SUP Stroke Technique.

Clark-Carter D. 2018. Quantitative Psychological Research : The Complete Student's Companion. Milton: Routledge.

Cohen J. 2013. Statistical power analysis for the behavioral sciences. Burlington: Elsevier Science.

Dionne M. 2014. Stand Up Paddleboarding: Paddling Technique.

Fiore DC, and Houston JD. 2001. Injuries in whitewater kayaking. British Journal of Sports Medicine 35:235. 10.1136/bjsm.35.4.235

Fleming N, Donne B, Fletcher D, and Mahony N. 2012. A biomechanical assessment of ergometer task specificity in elite flatwater kayakers. Journal of Sports Science \& Medicine 11:16-25.

Furness J, Olorunnife O, Schram B, Climstein M, and Hing W. 2017. Epidemiology of injuries in standup paddle boarding. Orthopaedic Journal of Sports Medicine (5) 6.

Gomes BB, Ramos NV, Conceicao FA, Sanders RH, Vaz MA, and Vilas-Boas JP. 2015. Paddling Force Profiles at Different Stroke Rates in Elite Sprint Kayaking. Journal of Applied Biomechanics 31:258-263. 10.1123/jab.2014-0114

Hagemann G, Rijke AM, and Mars M. 2004. Shoulder Pathoanatomy in Marathon Kayakers. British Journal of Sports Medicine 38:413-417.

Peer) reviewing PDF | (2019:05:37238:1:1:NEW 3 Oct 2019) 
299

300

301

302

303

304

305

306

307

308

309

310

311

312

313

314

315

316

317

318

319

320

321

322

Ho SR, Smith R, and Meara D. 2009. Biomechanical analysis of dragon boat paddling: A comparison of elite and sub-elite paddlers. Journal of Sports Sciences 27:37-47. 10.1080/02640410802491350

Kendal SJ, and Sanders RH. 1992. The Technique of Elite Flatwater Kayak Paddlers Using the Wing Paddle. International Journal of Sport Biomechanics 8:233-250. 10.1123/ijsb.8.3.233

Limonta E, Squadrone R, Rodano R, Marzegan A, Veicsteinas A, Merati G, and Sacchi M. 2010a. Tridimensional kinematic analysis on a kayaking simulator: key factors to successful performance. Sport Sciences for Health 6:27-34. 10.1007/s11332-010-0093-7

Limonta E, Squadrone R, Rodano R, Marzegan A, Veicsteinas A, Merati G, and Sacchi M. 2010b. Tridimensional kinematic analysis on a kayaking simulator: key factors to successful performance. Official Journal of the Faculty of Exercise Sciences - University of Milan 6:27-34. $10.1007 / \mathrm{s} 11332-010-0093-7$

Mei-Dan O, and Carmont MR. 2013. Adventure and extreme sports injuries: Epidemiology, treatment, rehabilitation and prevention.

Schram B. 2015. Stand up paddle boarding : an analysis of a new sport and recreational activity PhD Thesis. Bond University.

Schram B, Hing W, and Climstein M. 2015. Profiling the sport of stand- up paddle boarding. Journal of Sports Sciences:1-8. 10.1080/02640414.2015.1079331

Schram B, Hing W, and Climstein M. 2016a. Laboratory-and field-based assessment of maximal aerobic power of elite stand-up paddle-board athletes. International Journal of Sports Physiology and Performance 11:28-32. 10.1123/ijspp.2015-0076

Schram B, Hing W, and Climstein M. 2016b. The physiological, musculoskeletal and psychological effects of stand up paddle boarding. BMC Sports Science, Medicine and Rehabilitation 8:32. $10.1186 / \mathrm{s} 13102-016-0057-6$

Schram B, Hing W, and Climstein M. 2016c. Profiling the sport of stand-up paddle boarding. Journal of Sports Sciences 34:937-944. 10.1080/02640414.2015.1079331 
324 Schram B, Hing W, and Climstein M. 2017. The Long-Term Effects of Stand-up Paddle Boarding: A $325 \quad$ Case Study. International Journal of Sports and Exercise Medicine 3:065.

326 Stehlik R. 2011. Paddle Technique Part 2 - The Three Ingredients of a Powerful Stroke.

327 Wassinger CA, Myers JB, Sell TC, Oyama S, Rubenstein EN, and Lephart SM. 2011. Scapulohumeral 328 kinematic assessment of the forward kayak stroke in experienced whitewater kayakers. Sports Biomechanics 10:98-109. 10.1080/14763141.2011.569563

330 Wordpress. 2015. SUP Paddle Technique.

331 Zahalka F, Maly T, Mala L, Doktor M, and Vetrovsky J. 2011. Kinematic analysis of canoe stroke and its changes during different types of paddling pace - case study. Journal of Human Kinetics 29:2533. $10.2478 / \mathrm{v} 10078-011-0036-7$ 
Figure 1

A graphical representation of the differences in stroke kinematics between experienced and inexperienced participants.

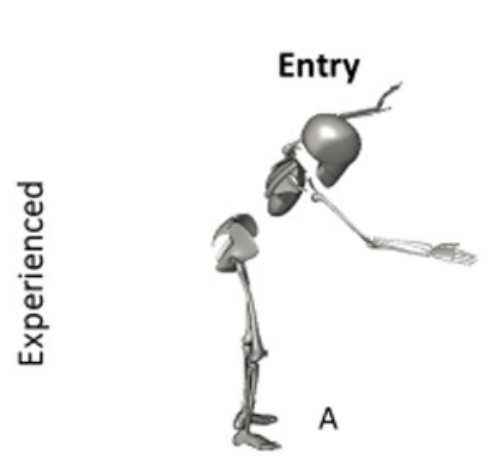

\section{Stroke phase}
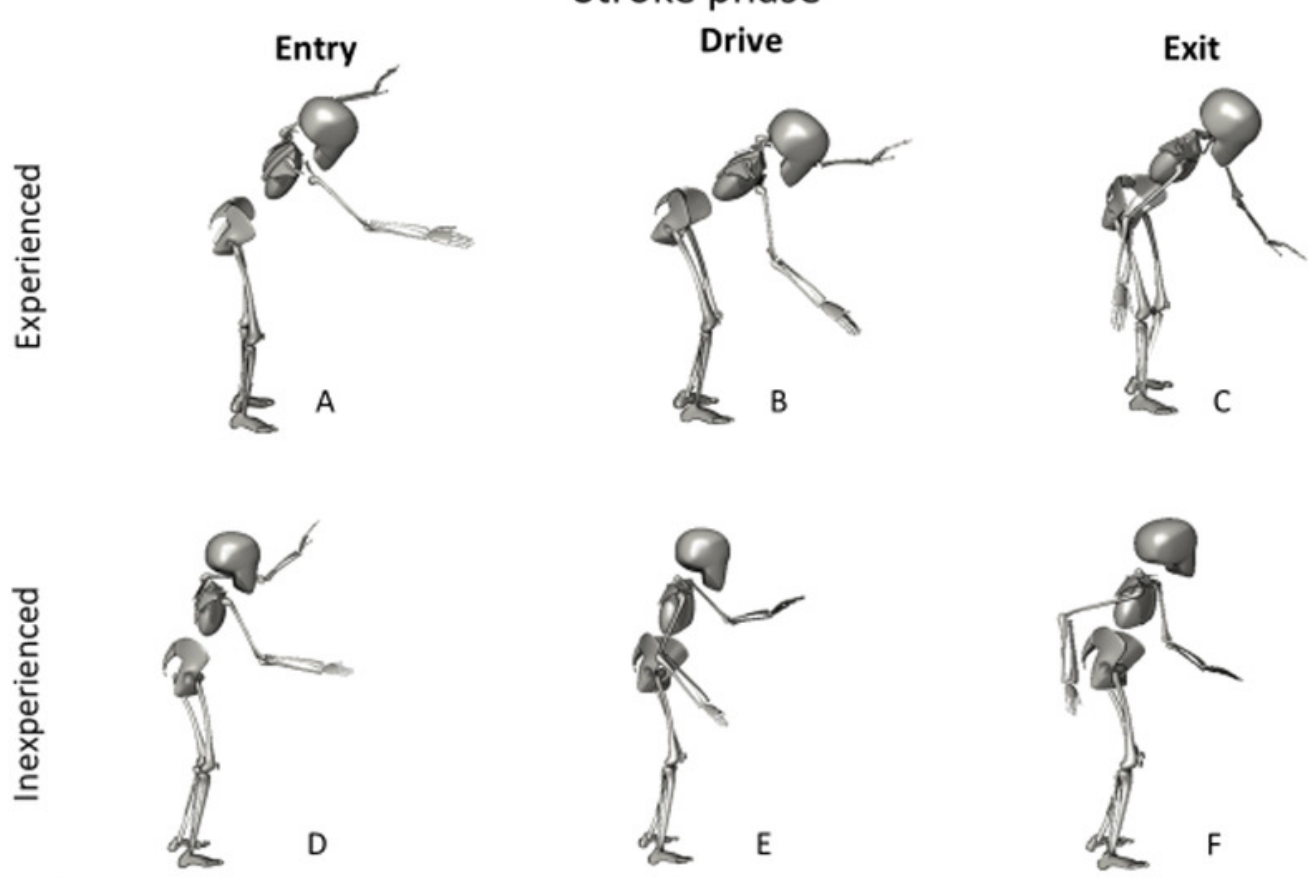


\section{Table 1 (on next page)}

Motion comparison at selected joints between inexperienced and inexperienced paddlers.

Results reported as average range \pm SD. 
1

\begin{tabular}{cccccc}
\hline Movement & Variable & Inexperienced & Experienced & $\boldsymbol{U}$ & Effect Size \\
\hline Shoulder Flexion & ROM & $78.9 \pm 24.9$ & $56.6 \pm 17.3$ & $0.010^{*}$ & -0.49 \\
& Max & $114.1 \pm 23.5$ & $131.5 \pm 9.0$ & 0.073 & -0.36 \\
& Min & $35.2 \pm 28.5$ & $74.9 \pm 16.3$ & $0.001^{*}$ & -0.62 \\
\hline Elbow Flexion & ROM & $47.1 \pm 22.0$ & $47.7 \pm 18.6$ & 0.910 & -0.28 \\
& Max & $68.5 \pm 24.6$ & $53.7 \pm 21.7$ & 0.152 & -0.29 \\
& Min & $24.8 \pm 13.5$ & $6.0 \pm 9.2$ & $0.000^{*}$ & -0.65 \\
\hline Trunk Flexion & ROM & $5.4 \pm 1.8$ & $5.7 \pm 1.6$ & 0.572 & -0.12 \\
& Max & $10.6 \pm 8.3$ & $12.18 \pm 5.9$ & 0.534 & -0.13 \\
& Min & $5.2 \pm 7.6$ & $6.48 \pm 6.5$ & 0.497 & -0.14 \\
\hline Trunk Abduction & ROM & $9.3 \pm 4.0$ & $6.8 \pm 1.8$ & 0.055 & -0.38 \\
& Max & $3.6 \pm 3.6$ & $1.7 \pm 4.9$ & 0.169 & -0.28 \\
& Min & $-5.7 \pm 3.8$ & $-5.1 \pm 5.8$ & 1.000 & -0.005 \\
\hline Trunk Rotation & ROM & $43.4 \pm 10.2$ & $39.9 \pm 9.8$ & 0.427 & -0.16 \\
& Max & $23.1 \pm 10.0$ & $19.1 \pm 6.4$ & 0.209 & -0.25 \\
& Min & $-20.4 \pm 9.8$ & $-20.8 \pm 7.3$ & 0.910 & -0.02 \\
\hline Hip Flexion & ROM & $50.0 \pm 18.5$ & $66.4 \pm 11.8$ & $0.035^{*}$ & -0.41 \\
& Max & $130.5 \pm 14.9$ & $134.2 \pm 8.9$ & 0.692 & -0.09 \\
& Min & $80.6 \pm 22.0$ & $67.8 \pm 6.8$ & 0.055 & -0.38 \\
\hline
\end{tabular}

2 